\title{
Evaluation of the optical conductivity tensor in terms of contour integrations
}

\author{
László Szunyogh†† and Peter Weinbergerł \\ $\dagger$ Department of Theoretical Physics, Technical University of Budapest, Budafoki út 8, 1521 \\ Budapest, Hungary \\ \$Center for Computational Materials Science, Vienna, Getreidemarkt 9/158, 1060 Wien, Austria
}

Received 16 June 1999

\begin{abstract}
For the case of finite lifetime broadening the standard Kubo formula for the optica conductivity tensor is rederived in terms of Green's functions by using contour integrations, whereby finite temperatures are accounted for by using the Fermi-Dirac distribution function. For zero lifetime broadening, the present formalism is related to expressions well known in the literature. Numerical aspects of how to calculate the corresponding contour integrals are also outlined.
\end{abstract}

\section{Introduction}

A general theory of linear response functions was formulated several decades ago [1-3] that became the basis of numerous studies of correlation functions, susceptibilities and transport properties in solid matter. Although early attempts have already been devoted within this theoretical framework to magneto-optical phenomena, i.e., the Faraday and the Kerr effect [2,4], it was the rapid development of both band-structure methods and computational facilities that brought about the renaissance in this field from the end of the 1980s (see [5,6] and references therein). This progress was built on the assumption that the original expression for the linear response functions, derived on the basis of a non-relativistic approach, is still valid when applying relativistic band-structure methods. Recently, this has been rigorously confirmed by Huhne and Ebert [7].

Within an independent-particle picture the interband part of the optical conductivity tensor at finite temperatures, $\Sigma_{\mu \nu}(\omega)$, is given by $[1,3]$

$$
\Sigma_{\mu \nu}(\omega)=\frac{\hbar}{\mathrm{i} V} \sum_{m, n} \frac{f\left(\epsilon_{n}\right)\left(1-f\left(\epsilon_{m}\right)\right)}{\epsilon_{n}-\epsilon_{m}}\left\{\frac{J_{n m}^{\mu} J_{m n}^{\nu}}{\hbar \omega+\epsilon_{n}-\epsilon_{m}+\mathrm{i} \delta}+\frac{J_{n m}^{\nu} J_{m n}^{\mu}}{\hbar \omega+\epsilon_{m}-\epsilon_{n}+\mathrm{i} \delta}\right\}
$$

where $\omega$ is the frequency, $f(\epsilon)$ is the Fermi function, $V$ is the volume of the system and the $J_{n m}^{\mu}=\left\langle n\left|J^{\mu}\right| m\right\rangle$ are the matrix elements of the current-density operator $J^{\mu}(\mu=x, y, z)$ with respect to the eigenfunctions $|n\rangle$ of the one-electron Hamiltonian corresponding to energy eigenvalues $\epsilon_{n}$. Lifetime-broadening effects due to different scattering processes are partially accounted for by a finite value of $\delta=\hbar / \tau \sim 0.03 \mathrm{Ryd}$, where $\tau$ can be associated with a relaxation time $[5,6]$. Such a lifetime effect can be incorporated naturally into linear response theory either by assuming an adiabatic switching on of the external field $\phi(r, t)=\phi(r) \mathrm{e}^{t / \tau}[8]$ or, equivalently, by convoluting the corresponding frequency-dependent conductivity for $\delta \rightarrow+0$ with a Lorentzian of finite halfwidth $\delta$. 
Expression (1) is usually directly evaluated by applying standard band-structure methods for ordered bulk (three-dimensional translationally invariant) systems by associating the states $|n\rangle$ with (three-dimensional) Bloch functions [2,5]. This kind of approach, however, cannot be extended easily to layered systems or systems with a surface, i.e., to systems with (at best) two-dimensional translational symmetry. Furthermore, it is totally unsuited to dealing with disorder such as interdiffusion effects in magnetic multilayer systems.

For zero lifetime broadening an alternative approach consists of splitting equation (1) into an absorptive and a dispersive part, which in turn are interrelated via the KramersKronig relations $[4,6]$. Thus it is sufficient to directly calculate the absorptive part only, the corresponding Kubo formula of which can be expressed in terms of Green's functions as was shown by Butler [9] for $\omega=0$, and which was used recently by Banhart [10] for $\omega>0$. Even much earlier, Luttinger [8] derived a general formula for the conductivity tensor which builds on the Green's function of the system. However, when using this method the corresponding energy integrals have to be taken along the real axis which makes a calculation of $\Sigma_{\mu \nu}(\omega)$ enormously tedious and also numerically hard to control.

In the present work a suitable extension of Luttinger's formula is given in terms of contour integrations that avoids the above-mentioned numerical difficulties, and is suitable for applications to magnetic multilayer systems and for treating alloying effects (interdiffusion etc) by means of the coherent potential approximation (CPA).

\section{Contour integrations}

Following Luttinger [8], equation (1) can be rewritten as

$$
\Sigma_{\mu \nu}(\omega)=\frac{\sigma_{\mu \nu}(\zeta)-\sigma_{\mu \nu}(0)}{\zeta}
$$

where $\zeta=\omega+\mathrm{i} \delta / \hbar$ and

$$
\sigma_{\mu \nu}(\zeta)=\frac{\mathrm{i}}{V} \sum_{m, n} \frac{f\left(\epsilon_{n}\right)-f\left(\epsilon_{m}\right)}{\hbar \zeta+\epsilon_{n}-\epsilon_{m}} J_{n m}^{\mu} J_{m n}^{\nu} .
$$

For simplicity, in what follows, we denote $\sigma_{\mu \nu}(\zeta)$ as $\sigma_{\mu \nu}(\omega)$ [11], in whose calculation we will be careful to keep it in mind that we have a finite imaginary part of the denominator in equation (3). Consider a pair of eigenvalues $\epsilon_{n}$ and $\epsilon_{m}$. For a suitable contour $\mathcal{C}$ in the complex-energy plane (see figure 1) the residue theorem implies

$$
\begin{aligned}
\oint_{\mathcal{C}} \mathrm{d} z \frac{f(z)}{\left(z-\epsilon_{n}\right)\left(z-\epsilon_{m}+\hbar \omega+\mathrm{i} \delta\right)} \\
=-2 \pi \mathrm{i} \frac{f\left(\epsilon_{n}\right)}{\epsilon_{n}-\epsilon_{m}+\hbar \omega+\mathrm{i} \delta}+2 \mathrm{i} \delta_{T} \sum_{k=-N_{2}+1}^{N_{1}} \frac{1}{\left(z_{k}-\epsilon_{n}\right)\left(z_{k}-\epsilon_{m}+\hbar \omega+\mathrm{i} \delta\right)}
\end{aligned}
$$

where the $z_{k}=\epsilon_{F}+\mathrm{i}(2 k-1) \delta_{T}\left(\epsilon_{F}\right.$ is the Fermi energy, $k_{B}$ the Boltzmann constant, $T$ the temperature and $\delta_{T}=\pi k_{B} T$ ) are the so-called Matsubara poles. In equation (4) it was supposed that the $N_{1}$ and $N_{2}$ Matsubara poles in the upper and lower semi-plane lie within the contour $\mathcal{C}$, respectively, i.e.,

$$
\begin{aligned}
& \left(2 N_{1}-1\right) \delta_{T}<\delta_{1}<\left(2 N_{1}+1\right) \delta_{T} \\
& \left(2 N_{2}-1\right) \delta_{T}<\delta_{2}<\left(2 N_{2}+1\right) \delta_{T} .
\end{aligned}
$$


Equation (4) can be rearranged as

$$
\begin{aligned}
\mathrm{i} \frac{f\left(\epsilon_{n}\right)}{\hbar \omega+\epsilon_{n}-\epsilon_{m}+\mathrm{i} \delta}=-\frac{1}{2 \pi} \oint_{\mathcal{C}} \mathrm{d} z \frac{f(z)}{\left(z-\epsilon_{n}\right)\left(z-\epsilon_{m}+\hbar \omega+\mathrm{i} \delta\right)} \\
+\mathrm{i} \frac{\delta_{T}}{\pi} \sum_{k=-N_{2}+1}^{N_{1}} \frac{1}{\left(z_{k}-\epsilon_{n}\right)\left(z_{k}-\epsilon_{m}+\hbar \omega+\mathrm{i} \delta\right)} .
\end{aligned}
$$

Similarly, by choosing a contour $\mathcal{C}^{\prime}$ as shown in figure 2, the following expression:

$$
\begin{array}{r}
-\mathrm{i} \frac{f\left(\epsilon_{m}\right)}{\hbar \omega+\epsilon_{n}-\epsilon_{m}+\mathrm{i} \delta}=\frac{1}{2 \pi} \oint_{\mathcal{C}^{\prime}} \mathrm{d} z \frac{f(z)}{\left(z-\epsilon_{m}\right)\left(z-\epsilon_{n}-\hbar \omega-\mathrm{i} \delta\right)} \\
+\mathrm{i} \frac{\delta_{T}}{\pi} \sum_{k=-N_{1}+1}^{N_{2}} \frac{1}{\left(z_{k}-\epsilon_{m}\right)\left(z_{k}-\epsilon_{n}-\hbar \omega-\mathrm{i} \delta\right)}
\end{array}
$$

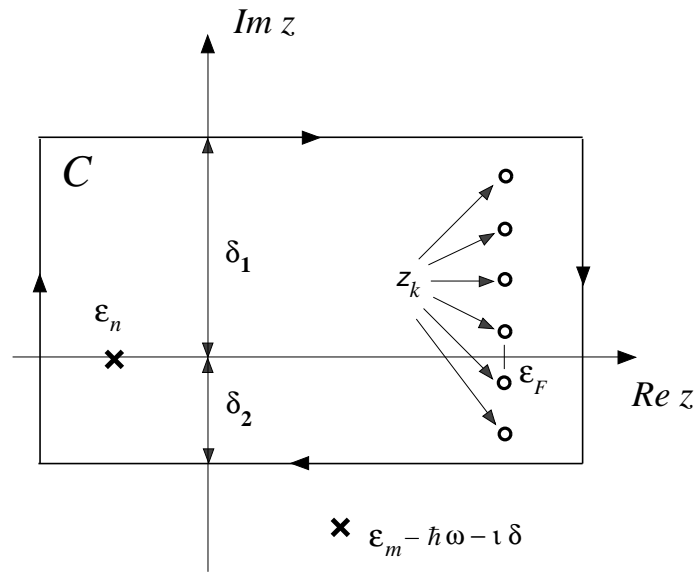

Figure 1. The contour in the complex plane corresponding to the integration in equation (4).

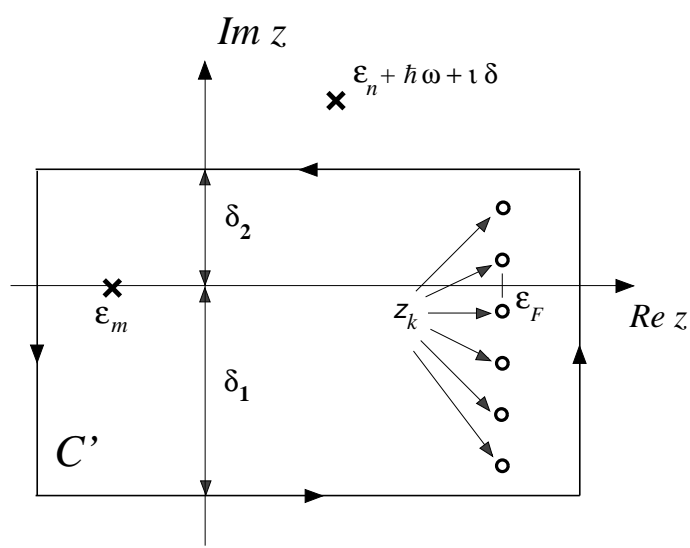

Figure 2. The contour in the complex plane corresponding to the integration in equation (8). 
can be derived. On inserting equations (7) and (8) into equation (3) and closing the contours at $\infty$ and $-\infty, \sigma_{\mu \nu}(\omega)$ is given by

$$
\begin{aligned}
\sigma_{\mu \nu}(\omega)=-\frac{1}{2 \pi V} & \left\{\oint_{\mathcal{C}} \mathrm{d} z f(z) \sum_{m, n} \frac{J_{n m}^{\mu} J_{m n}^{\nu}}{\left(z-\epsilon_{n}\right)\left(z-\epsilon_{m}+\hbar \omega+\mathrm{i} \delta\right)}\right. \\
& \left.-\oint_{\mathcal{C}^{\prime}} \mathrm{d} z f(z) \sum_{m, n} \frac{J_{n m}^{\mu} J_{m n}^{\nu}}{\left(z-\epsilon_{m}\right)\left(z-\epsilon_{n}-\hbar \omega-\mathrm{i} \delta\right)}\right\} \\
& +\mathrm{i} \frac{\delta_{T}}{\pi V}\left\{\sum_{k=-N_{2}+1}^{N_{1}} \sum_{m, n} \frac{J_{n m}^{\mu} J_{m n}^{\nu}}{\left(z_{k}-\epsilon_{n}\right)\left(z_{k}-\epsilon_{m}+\hbar \omega+\mathrm{i} \delta\right)}\right. \\
& \left.+\sum_{k=-N_{1}+1}^{N_{2}} \sum_{m, n} \frac{J_{n m}^{\mu} J_{m n}^{\nu}}{\left(z_{k}-\epsilon_{m}\right)\left(z_{k}-\epsilon_{n}-\hbar \omega-\mathrm{i} \delta\right)}\right\} .
\end{aligned}
$$

It is now straightforward to rewrite equation (9) in terms of the resolvent [12]:

$$
G(z)=\sum_{n} \frac{|n\rangle\langle n|}{z-\epsilon_{n}}
$$

such that

$$
\begin{aligned}
\sigma_{\mu \nu}(\omega)=-\frac{1}{2 \pi V} & \left\{\oint_{\mathcal{C}} \mathrm{d} z f(z) \operatorname{Tr}\left[J^{\mu} G(z+\hbar \omega+\mathrm{i} \delta) J^{\nu} G(z)\right]\right. \\
& \left.-\oint_{\mathcal{C}^{\prime}} \mathrm{d} z f(z) \operatorname{Tr}\left[J^{\mu} G(z) J^{\nu} G(z-\hbar \omega-\mathrm{i} \delta)\right]\right\} \\
& +\mathrm{i} \frac{\delta_{T}}{\pi V}\left\{\sum_{k=-N_{2}+1}^{N_{1}} \operatorname{Tr}\left[J^{\mu} G\left(z_{k}+\hbar \omega+\mathrm{i} \delta\right) J^{v} G\left(z_{k}\right)\right]\right. \\
& \left.+\sum_{k=-N_{1}+1}^{N_{2}} \operatorname{Tr}\left[J^{\mu} G\left(z_{k}\right) J^{v} G\left(z_{k}-\hbar \omega-\mathrm{i} \delta\right)\right]\right\}
\end{aligned}
$$

where Tr denotes the trace of an operator. By using the quantity given below, originally introduced by Butler [9]:

$$
\tilde{\sigma}_{\mu \nu}\left(z_{1}, z_{2}\right)=-\frac{1}{2 \pi V} \operatorname{Tr}\left[J^{\mu} G\left(z_{1}\right) J^{\nu} G\left(z_{2}\right)\right]
$$

for which the following symmetry relations apply:

$$
\begin{aligned}
& \tilde{\sigma}_{\nu \mu}\left(z_{2}, z_{1}\right)=\tilde{\sigma}_{\mu \nu}\left(z_{1}, z_{2}\right) \\
& \tilde{\sigma}_{\mu \nu}\left(z_{1}^{*}, z_{2}^{*}\right)=\tilde{\sigma}_{\nu \mu}\left(z_{1}, z_{2}\right)^{*}=\tilde{\sigma}_{\mu \nu}\left(z_{2}, z_{1}\right)^{*}
\end{aligned}
$$

$\sigma_{\mu \nu}(\omega)$ can be written as

$$
\begin{aligned}
\sigma_{\mu \nu}(\omega)=\oint_{\mathcal{C}} \mathrm{d} z f(z) \widetilde{\sigma}_{\mu \nu}(z+\hbar \omega+\mathrm{i} \delta, z)-\oint_{\mathcal{C}^{\prime}} \mathrm{d} z f(z) \widetilde{\sigma}_{\mu \nu}(z, z-\hbar \omega-\mathrm{i} \delta) \\
\quad-2 \mathrm{i} \delta_{T}\left\{\sum_{k=-N_{2}+1}^{N_{1}} \tilde{\sigma}_{\mu \nu}\left(z_{k}+\hbar \omega+\mathrm{i} \delta, z_{k}\right)+\sum_{k=-N_{1}+1}^{N_{2}} \widetilde{\sigma}_{\mu \nu}\left(z_{k}, z_{k}-\hbar \omega-\mathrm{i} \delta\right)\right\}
\end{aligned}
$$


which, because of the reflection symmetry for the contours $\mathcal{C}$ and $\mathcal{C}^{\prime}$ (see figures 1 and 2) and the relations in equation (13), can be transformed to

$$
\begin{array}{r}
\sigma_{\mu \nu}(\omega)=\oint_{\mathcal{C}} \mathrm{d} z f(z) \widetilde{\sigma}_{\mu \nu}(z+\hbar \omega+\mathrm{i} \delta, z)-\left(\oint_{\mathcal{C}} \mathrm{d} z f(z) \widetilde{\sigma}_{\mu \nu}(z-\hbar \omega+\mathrm{i} \delta, z)\right)^{*} \\
-2 \mathrm{i} \delta_{T} \sum_{k=-N_{2}+1}^{N_{1}}\left\{\widetilde{\sigma}_{\mu \nu}\left(z_{k}+\hbar \omega+\mathrm{i} \delta, z_{k}\right)+\widetilde{\sigma}_{\mu \nu}\left(z_{k}-\hbar \omega+\mathrm{i} \delta, z_{k}\right)^{*}\right\} .
\end{array}
$$

Equation (15) displays the central result of the present work: it compactly expresses the optical conductivity tensor in terms of contributions of a contour integral and those due to Matsubara poles. The quantities occurring of the type $\widetilde{\sigma}_{\mu \nu}\left(z_{1}, z_{2}\right)$ are now exactly of the form for which substitutional disorder (CPA) for layered systems $[13,14]$ can be treated. It should be noted that in the case of site-diagonal terms-see [14] —in principle for the evaluation of the contour integrals the 'irregular' part of the Green's function also has to be included. Since the inhomogeneous CPA for semi-infinite systems and the corresponding Kubo-Greenwood approach to electric transport are discussed at length in [14], no further discussion with respect to layered systems is needed.

\section{Integration along the real axis: the limit of zero lifetime broadening}

In this section we give the relationship of equation (15) to formulations existing in the literature. For this reason the contour $\mathcal{C}$ is deformed to the real axis such that the contributions from the Matsubara poles vanish. On using the relations in equation (13), equation (15) reduces to

$$
\begin{aligned}
\sigma_{\mu \nu}(\omega)=\int_{-\infty}^{\infty} \mathrm{d} \epsilon f(\epsilon)\left[\widetilde{\sigma}_{\mu \nu}(\epsilon+\hbar \omega+\mathrm{i} \delta, \epsilon+\mathrm{i} 0)-\widetilde{\sigma}_{\mu \nu}(\epsilon+\hbar \omega+\mathrm{i} \delta, \epsilon-\mathrm{i} 0)\right] \\
\quad-\int_{-\infty}^{\infty} \mathrm{d} \epsilon f(\epsilon)\left[\widetilde{\sigma}_{\mu \nu}(\epsilon-\mathrm{i} 0, \epsilon-\hbar \omega-\mathrm{i} \delta)-\widetilde{\sigma}_{\mu \nu}(\epsilon+\mathrm{i} 0, \epsilon-\hbar \omega-\mathrm{i} \delta)\right] .
\end{aligned}
$$

That is,

$$
\begin{gathered}
\sigma_{\mu \nu}(\omega)=-\frac{1}{2 \pi V} \int_{-\infty}^{\infty} \mathrm{d} \epsilon f(\epsilon) \operatorname{Tr}\left\{J^{\mu} G(\epsilon+\hbar \omega+\mathrm{i} \delta) J^{\nu}\left\{G^{+}(\epsilon)-G^{-}(\epsilon)\right\}\right. \\
\left.+J^{\mu}\left\{G^{+}(\epsilon)-G^{-}(\epsilon)\right\} J^{\nu} G(\epsilon-\hbar \omega-\mathrm{i} \delta)\right\}
\end{gathered}
$$

where we have introduced upper and lower limits for the resolvents [12]. On taking the limit $\delta \rightarrow 0$, equation (17) becomes equivalent to equation (5.15) of reference [8] for $\boldsymbol{q}=0$ :

$$
\begin{gathered}
\sigma_{\mu \nu}(\omega)=-\frac{1}{2 \pi V} \int_{-\infty}^{\infty} \mathrm{d} \epsilon f(\epsilon) \operatorname{Tr}\left\{J^{\mu} G^{+}(\epsilon+\hbar \omega) J^{\nu}\left\{G^{+}(\epsilon)-G^{-}(\epsilon)\right\}\right. \\
\left.+J^{\mu}\left\{G^{+}(\epsilon)-G^{-}(\epsilon)\right\} J^{\nu} G^{-}(\epsilon-\hbar \omega)\right\} .
\end{gathered}
$$

Note that by shifting in the second term of equation (18) the argument of the integration by $\hbar \omega$, the Hermitian part of $\sigma_{\mu \nu}(\omega)$ can be expressed as

$$
\begin{aligned}
& \sigma_{1, \mu \nu}(\omega) \equiv \frac{1}{2}(\left.\sigma_{\mu \nu}(\omega)+\sigma_{v \mu}(\omega)^{*}\right)=-\frac{1}{4 \pi V} \int_{-\infty}^{\infty} \mathrm{d} \epsilon(f(\epsilon)-f(\epsilon+\hbar \omega)) \\
& \times \operatorname{Tr}\left\{J^{\mu}\left\{G^{+}(\epsilon+\hbar \omega)-G^{-}(\epsilon+\hbar \omega)\right\} J^{\nu}\left\{G^{+}(\epsilon)-G^{-}(\epsilon)\right\}\right\} .
\end{aligned}
$$

Since quite clearly $\sigma_{1, \mu \nu}(0)=0$, from equation (2) we get

$$
\Sigma_{1, \mu \nu}(\omega)=\sigma_{1, \mu \nu}(\omega) / \omega
$$

as used in reference [10]. 
In order to obtain the correct zero-frequency formula, one has to take first the $\omega \rightarrow 0$ limit of equation (16) and then, after insertion into equation (2), the $\delta \rightarrow 0$ limit has to be performed. Making use of the analyticity of the Green's functions in the upper and lower complex semi-planes, this leads to [16]

$$
\begin{aligned}
\Sigma_{\mu \nu}(0)=- & \frac{\hbar}{2 \pi V} \int_{-\infty}^{\infty} \mathrm{d} \epsilon f(\epsilon) \\
& \times \operatorname{Tr}\left\{J^{\mu} \frac{\partial G^{+}(\epsilon)}{\partial \epsilon} J^{\nu}\left[G^{+}(\epsilon)-G^{-}(\epsilon)\right]-J^{\mu}\left[G^{+}(\epsilon)-G^{-}(\epsilon)\right] J^{\mu} \frac{\partial G^{-}(\epsilon)}{\partial \epsilon}\right\}
\end{aligned}
$$

which can be integrated by parts. The corresponding expression at $T=0$ for the diagonal elements yields Butler's original formula [9]:

$\Sigma_{\mu \mu}(0)_{(T=0)}=-\frac{\hbar}{4 \pi V} \operatorname{Tr}\left\{J^{\mu}\left[G^{+}\left(\epsilon_{F}\right)-G^{-}\left(\epsilon_{F}\right)\right] J^{\mu}\left[G^{+}\left(\epsilon_{F}\right)-G^{-}\left(\epsilon_{F}\right)\right]\right\}$.

\section{Practical evaluation of the contour integrals}

Returning to equation (15) we are left with the task of evaluating the contour integrals that occur, in a manner suitable for computational purposes. This is particularly demanding because, in principle, integrations from $-\infty$ to $\infty$ are involved. Recalling figure 1 , it is essential to note that the integration paths can be chosen arbitrarily with the only constraint being that the one in the lower complex semi-plane should lie in the range $0>\operatorname{Im}(z)>-\delta$. It is trivial to show that, if the contributions from the core states are not considered, the contour $\mathcal{C}$ can be closed at $\epsilon_{b}$ (the bottom of the valence band) which lies energetically just below the regime of valence states. Consequently, the integrals in equation (15) can be split up as follows:

$$
\begin{aligned}
\oint_{\mathcal{C}} \mathrm{d} z f(z) \widetilde{\sigma}_{\mu \nu}(z \pm \hbar \omega+\mathrm{i} \delta, z) \\
\quad=\int_{\epsilon_{b}+\mathrm{i} 0}^{\infty+\mathrm{i} \delta_{1}} \mathrm{~d} z f(z) \widetilde{\sigma}_{\mu \nu}(z \pm \hbar \omega+\mathrm{i} \delta, z)-\int_{\epsilon_{b}-\mathrm{i} 0}^{\infty-\mathrm{i} \delta_{2}} \mathrm{~d} z f(z) \widetilde{\sigma}_{\mu \nu}(z \pm \hbar \omega+\mathrm{i} \delta, z) .
\end{aligned}
$$

Because of the fast decay of the Fermi function $f(z)$ for $\operatorname{Re}(z)>\epsilon_{F}$, the integrals on the rhs of equation (22) can be terminated at

$$
\epsilon_{u}=\epsilon_{F}+n k_{B} T \quad(n \simeq 5-10) .
$$

Finally, after manipulating the contributions from the Matsubara poles-see equation (15) - the optical conductivity (or rather the zero-wavenumber current-current correlation function [11]) can be written as

$$
\sigma_{\mu \nu}(\omega)=\sigma_{\mu \nu}^{1}(\omega)+\sigma_{\mu \nu}^{2}(\omega)+\sigma_{\mu \nu}^{3}(\omega)
$$

where

$\sigma_{\mu \nu}^{1}(\omega)=\int_{\epsilon_{b}+\mathrm{i} 0}^{\epsilon_{u}+\mathrm{i} \delta_{1}} \mathrm{~d} z f(z) \widetilde{\sigma}_{\mu \nu}(z+\hbar \omega+\mathrm{i} \delta, z)-\left(\int_{\epsilon_{b}+\mathrm{i} 0}^{\epsilon_{u}+\mathrm{i} \delta_{1}} \mathrm{~d} z f(z) \widetilde{\sigma}_{\mu \nu}(z-\hbar \omega+\mathrm{i} \delta, z)\right)^{*}$

and 
$\sigma_{\mu \nu}^{2}(\omega)=\int_{\epsilon_{b}-\mathrm{i} 0}^{\epsilon_{u}-\mathrm{i} \delta_{2}} \mathrm{~d} z f(z) \widetilde{\sigma}_{\mu \nu}(z+\hbar \omega+\mathrm{i} \delta, z)-\left(\int_{\epsilon_{b}-\mathrm{i} 0}^{\epsilon_{u}-\mathrm{i} \delta_{2}} \mathrm{~d} z f(z) \widetilde{\sigma}_{\mu \nu}(z-\hbar \omega+\mathrm{i} \delta, z)\right)^{*}$

and also

$$
\begin{aligned}
\sigma_{\mu \nu}^{3}(\omega)=-2 \mathrm{i} \delta_{T} & \sum_{k=N_{2}+1}^{N_{1}}\left\{\widetilde{\sigma}_{\mu \nu}\left(\epsilon_{F}+\hbar \omega+\mathrm{i}\left[\delta+(2 k-1) \delta_{T}\right], \epsilon_{F}+\mathrm{i}(2 k-1) \delta_{T}\right)\right. \\
+ & \left.\left.\widetilde{\sigma}_{\mu \nu}\left(\epsilon_{F}-\hbar \omega+\mathrm{i}\left[\delta+(2 k-1) \delta_{T}\right], \epsilon_{F}+\mathrm{i}(2 k-1) \delta_{T}\right)^{*}\right]\right\} \\
& -2 \mathrm{i} \delta_{T} \sum_{k=1}^{N_{2}}\left\{\widetilde{\sigma}_{\mu \nu}\left(\epsilon_{F}+\hbar \omega+\mathrm{i}\left[\delta+(2 k-1) \delta_{T}\right], \epsilon_{F}+\mathrm{i}(2 k-1) \delta_{T}\right)\right. \\
& +\widetilde{\sigma}_{\mu \nu}\left(\epsilon_{F}-\hbar \omega+\mathrm{i}\left[\delta+(2 k-1) \delta_{T}\right], \epsilon_{F}+\mathrm{i}(2 k-1) \delta_{T}\right)^{*} \\
& +\widetilde{\sigma}_{\mu \nu}\left(\epsilon_{F}+\hbar \omega+\mathrm{i}\left[\delta-(2 k-1) \delta_{T}\right], \epsilon_{F}-\mathrm{i}(2 k-1) \delta_{T}\right) \\
& \left.+\widetilde{\sigma}_{\mu \nu}\left(\epsilon_{F}-\hbar \omega+\mathrm{i}\left[\delta-(2 k-1) \delta_{T}\right], \epsilon_{F}-\mathrm{i}(2 k-1) \delta_{T}\right)^{*}\right\} .
\end{aligned}
$$

Preliminary calculations show that on choosing $\delta \simeq 0.05$ Ryd, $\delta_{1} \simeq 0.1-0.15$ Ryd and using Gaussian quadrature, a total of 30-100 energy points (depending on $\hbar \omega$ ) is sufficient to sample the above energy integrals. Furthermore, since most of these energy points lie either below the valence band or far enough away from the real axis (the energy point closest to the real axis is at $z_{1}=\epsilon_{F}+\mathrm{i} \delta_{T}$, where for $T=300 \mathrm{~K}, \delta_{T} \simeq 6 \mathrm{mRyd}$ ), the Brillouin-zone integrals occurring (see reference [14]) can be evaluated by using a sufficiently small set of $\boldsymbol{k}$-points in the surface Brillouin zone, thus ensuring excellent numerical stability.

\section{Summary}

In the present paper, by using a finite lifetime broadening (or a finite adiabatic switch-on parameter) we reformulated the expression for the (linear response) optical conductivity tensor in terms of contour integrations such that Green's functions (resolvents) naturally appear and finite temperatures are represented by the Fermi-Dirac distribution function. For zero lifetime broadening the traditional expressions for the conductivity tensor in terms of the Green's function are recovered. Finally, a scheme for calculating the contour integrals that occur, in a numerically efficient way, is suggested. Although primarily applications to $a b$ initio calculations of magneto-optical properties of ordered and disordered thin films in terms of the fully relativistic spin-polarized screened Korringa-Kohn-Rostoker method [14, 15] are currently under way, it should be noted that the present method can also be used to calculate other linear response functions, just as readily as it can easily be extended to the evaluation of non-linear response functions.

\section{Acknowledgments}

The authors are especially grateful to Professor B L Györffy for many stimulating discussions. This paper resulted from a collaboration partially funded by the Research and Technological Cooperation between Austria and Hungary (OMFB-Bundesministerium für Auswärtige Angelegenheiten, Contract No A-35/98). Financial support was provided also by the Austrian Science Foundation (Contract No P12146 and Contract No P12352), and the Hungarian National Science Foundation (Contract No OTKA T024137 and Contract No OTKA T030240). 


\section{References}

[1] Kubo R 1957 J. Phys. Soc. Japan 12570

[2] Wang C S and Callaway J 1974 Phys. Rev. B 94897

[3] Callaway J 1974 Quantum Theory of the Solid State part B (New York: Academic)

[4] Bennett H S and Stern E A 1965 Phys. Rev. 137 A448

[5] Oppeneer P M and Antonov V N 1996 Spin-Orbit-Influenced Spectroscopies of Magnetic Solids ed H Ebert and G Schütz (Berlin: Springer) pp 29-47

[6] Ebert H 1996 Rep. Prog. Phys. 591665

[7] Huhne T and Ebert H 1999 Phys. Rev. B submitted

Here it is claimed that in a fully relativistic formalism the so-called diamagnetic contribution to the conductivity is not explicitly present.

[8] Luttinger J M 1967 Mathematical Methods in Solid State and Superfluid Theory ed R C Clark and G H Derrick (Edinburgh: Oliver and Boyd) pp 157-93

[9] Butler W H 1985 Phys. Rev. B 313260

[10] Banhart J 1999 Phys. Rev. Lett. 822139

[11] In fact, $\sigma_{\mu \nu}(\omega)=\mathrm{i} \Pi_{\mu \nu}(\boldsymbol{q}=0, \omega)$, where $\Pi_{\mu \nu}(\boldsymbol{q} \omega)$ is the current-current correlation function. Compare with equation (3.8.9) of

Mahan G D 1990 Many-Particle Physics (New York: Plenum)

[12] Weinberger P 1990 Electron Scattering for Ordered and Disordered Matter (Oxford: Clarendon)

[13] Butler W H, Zhang X-G and Nicholson D M C 1994 J. Appl. Phys. 766808

Butler W H, Zhang X-G, Nicholson D M C and MacLaren J M 1995 Phys. Rev. B 5213399

[14] Weinberger P, Levy P M, Banhart J, Szunyogh L and Újfalussy B 1996 J. Phys.: Condens. Matter 87679

[15] Szunyogh L, Újfalussy B and Weinberger P 1995 Phys. Rev. B 519552

[16] Smrčka L and Středa P 1977 J. Phys. C: Solid State Phys. 102153 